\title{
Identification of Random Dynamic Force Using an Improved Maximum Entropy Regularization Combined with a Novel Conjugate Gradient
}

\author{
ChunPing Ren, ${ }^{1}$ NengJian Wang, ${ }^{1}$ and ChunSheng Liu ${ }^{2}$ \\ ${ }^{1}$ College of Mechanical and Electrical Engineering, Harbin Engineering University, Harbin 150001, China \\ ${ }^{2}$ School of Mechanical Engineering, Heilongjiang University of Science and Technology, Harbin 150022, China \\ Correspondence should be addressed to ChunPing Ren; renchunpin@sina.com
}

Received 2 May 2017; Revised 7 August 2017; Accepted 13 August 2017; Published 30 October 2017

Academic Editor: Xinkai Chen

Copyright (C) 2017 ChunPing Ren et al. This is an open access article distributed under the Creative Commons Attribution License, which permits unrestricted use, distribution, and reproduction in any medium, provided the original work is properly cited.

\begin{abstract}
We propose a novel mathematical algorithm to offer a solution for the inverse random dynamic force identification in practical engineering. Dealing with the random dynamic force identification problem using the proposed algorithm, an improved maximum entropy (IME) regularization technique is transformed into an unconstrained optimization problem, and a novel conjugate gradient (NCG) method was applied to solve the objective function, which was abbreviated as IME-NCG algorithm. The result of IMENCG algorithm is compared with that of ME, ME-CG, ME-NCG, and IME-CG algorithm; it is found that IME-NCG algorithm is available for identifying the random dynamic force due to smaller root mean-square-error (RMSE), lower restoration time, and fewer iterative steps. Example of engineering application shows that L-curve method is introduced which is better than Generalized Cross Validation (GCV) method and is applied to select regularization parameter; thus the proposed algorithm can be helpful to alleviate the ill-conditioned problem in identification of dynamic force and to acquire an optimal solution of inverse problem in practical engineering.
\end{abstract}

\section{Introduction}

The inverse problem, which is a typical mathematical problem, has been widely applied in many fields of practical engineering, such as vibration isolation, health monitoring, and fault diagnosis [1-3]. The primary concern of mathematical workers and engineering technicians in the inverse problem derives from the development of the theory of solving the problems of universal existence and ill-conditioned problems in practical engineering applications in the past few years [46]. Identification of dynamic force between pick and coalseam has been a major concern in failure analysis of rotary machinery; once accurate force sources are obtained, it is possible to apply some advanced algorithms to analyze the characteristics of the dynamic force signals. However, we can not obtain the accurate dynamic force directly by the traditional methods due to the ill-posedness of systems [79]. Aiming to reduce the above problems, it is necessary to obtain a method for solving ill-conditioned problem.

Accordingly, regularization techniques, which can treat the ill-conditioned problem, have been utilized in many fields [10-13]. In the past few years, maximum entropy (ME) regularization techniques have been put forward one after another, which has been applied successfully in the wide areas of image reconstruction, signal processing, force identification problem, and so on [14], and the advantages of using ME regularization technique are as follows: firstly, some important information from the incomplete data can be extracted; then the probability distribution of the constraint is hidden in it; and, finally, entropy term can be changed according to different objects [15]. Hence, ME regularization technique mentioned above can lay a firm foundation for the steady and accurate solutions of ill-conditioned problem. 
However, ME regularization technique is not perfect, which has been made better in many forms, and the means of improvement are generally concentrated in the entropy function combined with some optimization algorithms to solve regularization solution [16]. Mohammad-Djafari et al. [17] applied the improved entropy function combining the Lagrange method to process mass spectrometry data problems. Haffner et al. [18] discussed an improved maximum entropy modeling to handle with multiclass categorization problems using Newton iteration algorithm. In [19], by combining maximum entropy and Bayesian method, a new maximum entropy regularization is constructed to distribute force acting on boundary structure. Myrheim and Rue [20] presented a novel algorithm combining the ME method with the preconditioned conjugate gradient method to reconstruct image. In [21], the maximum entropy function and the conjugate gradient method were utilized, which can be used to solve absolute value equation problems. However, as for the improved entropy function mentioned, we consider that it is a numerical optimization algorithm, and its disadvantage is that the rate of convergence is not high, which is only suitable for simple objects, but the identified results are not ideal for complex objects. In fact, the entropy function can be redesigned according to the properties of different objects, and identification results of different objects can be achieved when we use different entropy functions combined with some different optimization algorithms [22].

In our study, a fast mathematical algorithm that can identify dynamic force between pick and coal-seam is determined. The technique we propose is new to our research field. Significant improvements are made in comparison with our previous work.

First, the force identification procedure is transformed into an unconstrained optimization problem due to being suboptimal for conventional regularization methods. In this work, a novel optimization mathematical algorithm, combining a novel improved maximum entropy (IME) regularization technique and a novel conjugate gradient (NCG) method (for short IME-NCG), is proposed to simplify the optimization process and improve the applicability of the algorithm.

Second, as a fast technique of IME-NCG, the optimized objective function is redesigned using the 1-norm estimation and the elongated entropy function in order to overcome the numerical instability.

Third, the optimal solution (stable solution) of inverse problem in practical engineering is studied. The technique proposed does not require any a priori information on the model for the random dynamic force identification. Only the displacement response measured by experiment is adequate for the identification algorithm.

Fourth, global convergence and stability of the solution for identified force model are proved to improve the precision and detailed information of random dynamic force identification.

Fifth, the L-curve criteria, combined with the IME regularization technique, are applied to select the suitable regularization parameter for dynamic force identification in complex vibration system.
Finally, the applicability of the proposed technique is effectively demonstrated through an engineering application example. It is found that the performance of random dynamic force identification using the combination of IME-NCG algorithm and L-curve is better than that of other previous works, which can effectively reduce the ill-posedness of random dynamic force identification.

In the present work, we aim at identifying random dynamic force between pick and coal-seam combining a novel improved maximum entropy (IME) regularization technique and a novel conjugate gradient (NCG) method. The structure of the paper is arranged as follows. In Section 2, the identification model of random dynamic force is established. In Section 3, the IME-NCG algorithm is proposed. In Section 4, we present the engineering application and results discussion. Some important conclusions are obtained in Section 5.

\section{Model of Identified Force}

An abridged general view of random dynamic force between pick and coal-seam structure is plotted in Figure 1. For deterministic MDOF structures, the kinetic equation can be expressed by the following form [23]:

$$
\begin{aligned}
& {[\mathbf{M}]\left\{\mathbf{y}^{\prime \prime}(t)\right\}+[\mathbf{C}]\left\{\mathbf{y}^{\prime}(t)\right\}+[\mathbf{K}]\{\mathbf{y}(t)\}} \\
& \quad=\{\mathbf{S}(x, y, z, t)\},
\end{aligned}
$$

where $\mathbf{M}, \mathbf{C}$, and $\mathbf{K}$ represent the mass matrix, damping matrix, and stiffness matrix, respectively; $\mathbf{S}(x, y, z, t)$ denotes the dynamic force column vector in different directions; $\mathbf{y}(t), \mathbf{y}^{\prime}(t)$, and $\mathbf{y}^{\prime \prime}(t)$ are displacement, velocity, and acceleration response vector, respectively.

The displacement of the structure can be described as

$$
\mathbf{y}(t)=\sum_{i=1}^{n} \boldsymbol{\varphi}_{i} q_{i}(t)=\mathbf{\Phi Q}
$$

where $\Phi$ is matrix of mode and $\Phi=\left[\boldsymbol{\varphi}_{1}, \boldsymbol{\varphi}_{2}, \ldots, \boldsymbol{\varphi}_{n}\right]$; $\mathbf{Q}$ is the displacement vector and time function in the generalized coordinates; $\mathbf{Q}=\left[q_{1}, q_{2}, \ldots, q_{n}\right]^{T}$.

By means of (1) and (2), the dynamic equations represented by the physical coordinates are transformed into the modal coordinate system, and the $n$ decoupled modal equations are obtained in the following form:

$$
q_{i}^{\prime \prime}+2 \xi_{i} \omega_{i} q_{i}^{\prime}+\omega_{i}^{2} q_{i}=\frac{\mathbf{F}_{i}(t)}{\mathbf{M}_{i}}
$$

in which $\xi_{i}$ is the $i$ order modal damping ratio; $\mathbf{M}_{i}, \mathbf{F}_{i}(t)$ are the generalized mass and the generalized force, respectively, and $\mathbf{M}_{i}=\boldsymbol{\varphi}_{i}^{T} \mathbf{M} \boldsymbol{\varphi}_{i}$ and $\mathbf{F}_{i}(t)=\boldsymbol{\varphi}_{i}^{T} \mathbf{S}(t)$.

The initial velocity and initial displacement of the system are assumed to be $\mathbf{x}_{0}^{\prime}$ and $\mathbf{x}_{0}$, respectively. So $q_{i}$ in (2) can be expressed as follows:

$$
q_{i}(t)=q_{1 i}(t)+q_{2 i}(t) .
$$




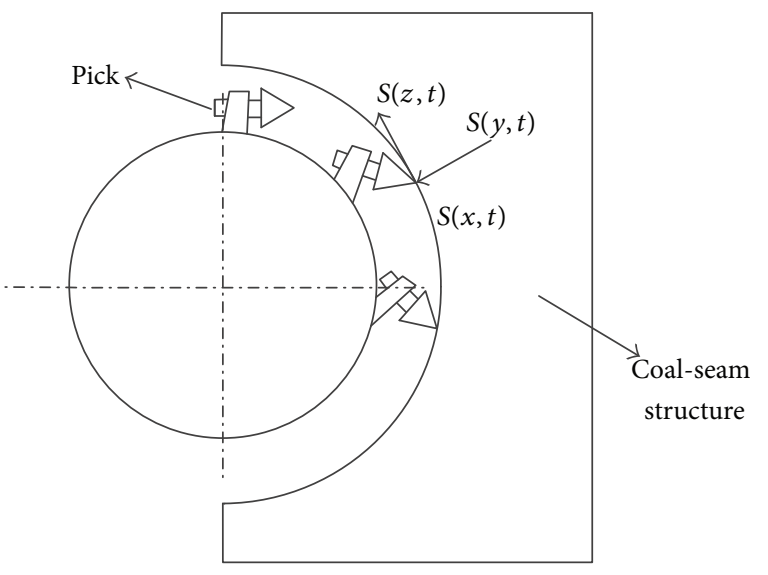

FIGURE 1: A schematic of dynamic forces between pick and coal-seam.

It is well known that $q_{1 i}(t)$ can be obtained by the homogeneous equation of (1), which can be described as follows [24]:

$$
\begin{array}{r}
q_{1 i}(t)=e^{-\xi_{i} \omega_{i} t}\left(q_{i 0}(t) \cos \left(\omega_{\mathrm{id}} t\right)\right. \\
\left.+\frac{q_{i 0}^{\prime}+\xi_{i} \omega_{i} q_{i 0}(t)}{\omega_{\mathrm{id}}} \sin \left(\omega_{\mathrm{id}} t\right)\right),
\end{array}
$$

where $q_{i 0}$ and $q_{i 0}^{\prime}$ denote the corresponding values in modal coordinates, which can be described as follows:

$$
q_{i 0}=\frac{\boldsymbol{\varphi}_{i}^{T} \mathbf{M} \mathbf{x}_{0}}{\mathbf{M}_{i}}
$$

$q_{2 i}$ can be expressed in the following form due to the fact it has nothing to do with the initial conditions of the system:

$$
q_{2 i}(t)=\frac{1}{M_{i} \omega_{\mathrm{id}}} \int_{0}^{t} F_{i}(\tau) e^{-\xi_{i} \omega_{i}(t-\tau)} \sin \omega_{\mathrm{id}}(t-\tau) d \tau,
$$
follows:

The displacement of the system can be expressed as

$$
\mathbf{y}=\mathbf{y}^{0}+\mathbf{y}^{*}
$$

in which $\mathbf{y}^{0}=\sum_{i=1}^{n} \varphi_{i} q_{1 i}$ and $\mathbf{y}^{*}=\sum_{i=1}^{n} \boldsymbol{\varphi}_{i} q_{2 i}$

The system force is identified according to the known displacement; the displacement is represented as follows:

$$
\mathbf{y}^{*}=\mathbf{y}-\mathbf{y}^{0}
$$

The following expressions can be obtained through (5) and (7) which are introduced into (9):

$$
y^{*}=\int_{0}^{t} \sum_{i=1}^{n} \frac{\boldsymbol{\varphi}_{i} \boldsymbol{\varphi}_{i}^{T}}{\mathbf{M}_{i} \omega_{\mathrm{id}}} \mathbf{S}_{i}(\tau) e^{-\xi_{i} \omega_{i}(t-\tau)} \sin \omega_{\mathrm{id}}(t-\tau) d \tau .
$$

In general, $\mathbf{y}^{*}(t)$ can be described as a convolution integral of the modal force and the corresponding Green kernel function:

$$
\mathbf{y}^{*}(t)=\int_{0}^{t} \mathbf{G}(t-\tau) \mathbf{S}(\tau) d \tau
$$

Hence, Green's kernel function can be described by comparing (10) and (11):

$$
\mathbf{G}(t)=\sum_{i=1}^{n} \frac{\boldsymbol{\varphi}_{i} \boldsymbol{\varphi}_{i}^{T}}{\mathbf{M}_{i} \omega_{\mathrm{id}}} e^{-\xi_{i} \omega_{i} t} \sin \omega_{\mathrm{id}} t .
$$

Let $\left\{\mathbf{y}^{*}(t)\right\}=\left\{y_{1}^{*}(t) \cdots y_{m}^{*}(t)\right\}^{T},\{\mathbf{S}(t)\}=\left\{S_{1}(t) \cdots S_{m}(t)\right\}^{T}$, and $\{\mathbf{G}(t)\}=\left\{g_{1}(t) \cdots g_{m}(t)\right\}^{T}$ then $t$ (11) was discretized, and it is transformed into a matrix form that can be expressed as follows:

$$
\left\{\begin{array}{c}
y_{1}^{*} \\
y_{2}^{*} \\
y_{3}^{*} \\
\vdots \\
y_{m}^{*}
\end{array}\right\}=\left[\begin{array}{ccccc}
g_{1} & 0 & 0 & \cdots & 0 \\
g_{2} & g_{1} & 0 & \cdots & 0 \\
g_{3} & g_{2} & g_{1} & \cdots & 0 \\
\vdots & \vdots & \vdots & \cdots & 0 \\
g_{m} & g_{m-1} & g_{m-2} & \cdots & 0
\end{array}\right]\left\{\begin{array}{c}
s_{1} \\
s_{2} \\
s_{3} \\
\vdots \\
s_{m}
\end{array}\right\},
$$

or simply noted as

$$
\mathbf{Y}=\mathbf{G S} \text {. }
$$

Owing to the responses containing large noise, so (14) is expressed by the following form:

$$
\mathbf{Y}^{c}=\mathbf{G S}_{\mathrm{id}}+e,
$$

where $\mathbf{S}_{\mathrm{id}}$ denotes the identified dynamic force, $e$ is the unknown noise, and $e=l \cdot \operatorname{std}(y(t)) \cdot \operatorname{rand}, l$ denotes a parameter level, $\operatorname{std}(\bullet)$ denotes the standard deviation, and rand denotes a random number which ranges from -1 to 1 . 
To identify $\mathbf{S}_{\mathrm{id}}$, we need to get $\mathbf{Y}^{c}$ and $\mathbf{G}$; moreover, it was found that (15) is a typical ill-conditioned problem. In some cases, small varieties in the right-hand side $e$ of (15) may lead to dramatic varieties in the solution [25-28]. In other words, they are very susceptible to error, in the inverse problem, this may directly lead to greater deviations of the identified results [29-31], and thus it is not realistic to solve (15) using traditional mathematical methods [32-34].

\section{Algorithm}

From the above analysis it can be seen that identification problem of dynamic force is ill-conditioned, whose solution is usually astaticism [35-38]. Therefore, it is necessary to find a way to achieve a stable solution. The regularization techniques are good method to alleviate the ill-conditioned problem. In the past few years, maximum entropy (ME) regularization techniques have been put forward one after another, which have been applied successfully in the wide area of practical engineering problem [39]. However, ME regularization technique is not very perfect; it has some disadvantages. Hence, in this work, an improved maximum entropy (IME) regularization technique is studied.

3.1. Maximum Entropy (ME) Regularization Method. It is generally known that maximum entropy (ME) regularization technique has been widely adopted in the past few years. The idea of the ME method is shown that the solving of (15) is transformed into that of an optimization problem [40]:

$$
\min J\left(\mathbf{S}_{\mathrm{id}}\right)=\left\|\mathbf{G S}_{\mathrm{id}}-\mathbf{Y}^{c}\right\|^{2}+\lambda \sum_{i=1}^{n} \mathbf{S}_{i} \ln \mathbf{S}_{i}
$$

where $\lambda$ represents the regularization parameter.

Let $\Omega\left(\mathbf{S}_{i}\right)=\sum_{i=1}^{n} \mathbf{S}_{i} \ln \left(\mathbf{S}_{i}\right)$, and it is called the entropy item. Thus, (16) can be replaced by the following:

$$
\min J\left(\mathbf{S}_{\mathrm{id}}\right)=\left\|\mathbf{G} \mathbf{S}_{\mathrm{id}}-\mathbf{Y}^{c}\right\|^{2}+\lambda \Omega\left(\mathbf{S}_{i}\right) .
$$

3.2. An Improved Maximum Entropy (IME) Regularization Method. In some circumstances, such as complex identified objects, ME regularization technique is not very perfect, and it has some disadvantages of poor quality of identification. Hence, in this paper, the entropy item of ME regularization technique is redesigned using the 1-norm estimation and the elongated entropy function according to the ill-posedness characteristics of dynamic force identification, which was named as an improved maximum entropy (IME) regularization technique, was proposed to process the dynamic force.

In this section, a function is used to design the entropy item which is expressed as follows according to 1-norm estimation method:

$$
\begin{aligned}
& \Omega\left(\mathbf{S}_{i}\right) \\
& \quad=\sum_{i=1}^{n}\left(1+\ln \left(1+\left|\mathbf{S}_{i}\right|^{p_{i}}\right)\right) \ln \left(1+\ln \left(1+\left|\mathbf{S}_{i}\right|^{p_{i}}\right)\right),
\end{aligned}
$$

where $|\bullet|$ represents 1 norm and $0<p_{i}<2$.

And $|\mathbf{S}|$ can be expressed by [41]

$$
|\mathbf{S}| \approx\left(\mathbf{S}^{2}+\xi\right)^{1 / 2}
$$

Hence, (18) is described by

$$
\begin{gathered}
\Omega\left(\mathbf{S}_{i}\right) \approx \sum_{i=1}^{n}\left(1+\ln \left(1+\left(\mathbf{S}_{i}^{2}+\xi\right)^{p_{i} / 2}\right)\right) \\
\cdot \ln \left(1+\ln \left(1+\left(\mathbf{S}_{i}^{2}+\xi\right)^{p_{i} / 2}\right)\right),
\end{gathered}
$$

where $\xi$ denotes the predetermined parameter.

Hence, a novel improved entropy item for dynamic force identification can be obtained, and it can be described as follows:

$$
\min J\left(\mathbf{S}_{\mathrm{id}}\right)=\left\|\mathbf{G S}_{\mathrm{id}}-\mathbf{Y}^{c}\right\|^{2}+\lambda \sum_{i=1}^{n}\left(1+\ln \left(1+\left(\mathbf{S}_{i}^{2}+\xi\right)^{p_{i} / 2}\right)\right) \ln \left(1+\ln \left(1+\left(\mathbf{S}_{i}^{2}+\xi\right)^{p_{i} / 2}\right)\right)
$$

It is obvious that the proposed entropy item is different with that of $\mathrm{ME}$ regularization technique; it mainly considers the 1-norm estimation and uses a better entropy item to make regular solutions more stable, thus which may be more suitable for the force identification of complex objects.

3.3. Selection of the Regularization Parameter. In this paper, we discuss L-curve method and Generalized Cross Validation (GCV) due to the validity and the classical character in choosing the regularization parameter [35, 42].

According to the regularization theory, the regularization parameter $\lambda$ for an improved maximum entropy (IME) regularization plays an important role in applying the numerical experiment process. First, the L-curve criterion is introduced to select an optimal regularization parameter.

By reading the relevant literature, we know that the Lcurve criterion is a very powerful way for selecting a suitable value of the regularization parameter for many meaningful engineering and mathematical problems.

It is well known that the L-curve is represented as a log$\log$ plot $\left(\log \left\|G S-Y^{c}\right\|_{2}, \log \|S\|_{2}\right)$ of the norm of the residual versus the corresponding norm of a regularized solution.

The curve shows a typical " $L$ " shape, and the optimal value for the regularization parameter is considered to be the one that corresponds to the corner of the curve [43]. 
Typically, the GCV function can be defined in the following form [44]:

$$
\min \operatorname{GCV}(\lambda)=\frac{\left\|G S(\lambda)-Y^{c}\right\|_{2}^{2}}{\operatorname{tr}\left(I-G G(\lambda)^{I}\right)^{2}},
$$

where $\operatorname{tr}(\bullet)$ denotes trace of matrix.

3.4. Solving of Objective Function. Equation (21) is considered as a class of unconstrained optimization problems and a series of optimization algorithms, which can treat such unconstrained optimization problem. In this work, a novel conjugate gradient (NCG) method was proposed to minimize (21). It has always been known that iterative form of conjugate gradient (CG) method are described as follows [45]:

$$
S_{k+1}=S_{k}+\alpha_{k} h_{k}
$$

where $\alpha_{k}$ denotes the step length, which can be computed using standard Armijo search conditions

$$
\begin{aligned}
\alpha_{k}=\max & \left\{\rho^{j}, j=0,1,2, \ldots\right\}, \\
& f\left(S_{k}+\alpha_{k} h_{k}\right) \leq f\left(S_{k}\right)+\delta \alpha_{k} g_{k}^{T} h_{k},
\end{aligned}
$$

where $\rho \in(0,1)$ and $\delta \in(0,0.5)$.

$h_{k}$ denotes the search direction

$$
h_{k}= \begin{cases}-g_{k}, & \text { if } k=0 \\ -g_{k}+\beta_{k} h_{k-1}, & \text { if } k \geq 1\end{cases}
$$

where $g_{k}$ denotes the gradient of $J\left(S_{k}\right)$ at the point $S_{k}, g_{k}=$ $\nabla J\left(S_{k}\right)$, and $\beta_{k}$ is conjugate parameter.

A novel search direction $h_{k}^{\text {new }}$ based on $[46,47]$ PRP conjugate gradient method is determined as follows:

$$
h_{k}^{\text {new }}= \begin{cases}-g_{k}, & \text { if } k=0 \\ -g_{k}+\beta_{k}^{\text {PRP }} z_{k} h_{k-1}^{\text {new }}, & \text { if } k \geq 1\end{cases}
$$

where

$$
\begin{aligned}
z_{k} & =\min \left\{\theta_{k}, 1\right\}, \quad \theta_{k}=\frac{c_{1}\left\|g_{k-1}\right\|^{2}}{L_{k}\left\|h_{k-1}^{\text {new }}\right\|^{2}}, \\
L_{k} & =\max \left\{\frac{\left\|g_{k}-g_{k-1}\right\|}{\left\|v_{k-1}\right\|}, L_{k-1}\right\}, \quad c_{0} \in(0,1), \\
v_{k-1} & =\alpha_{k-1} h_{k-1}^{\text {new }}, \quad L_{0}>0, \\
\beta_{k}^{\text {PRP }} & =\frac{g_{k}^{T}\left(g_{k}-g_{k-1}\right)}{\left\|g_{k-1}\right\|^{2}} .
\end{aligned}
$$

Algorithm 1.

Step 1. Given an initial point $S_{0}, c_{1} \in(0,1)$, and $L_{0}>0$, set $h_{0}=-g_{0}$ and $k=0$.

Step 2. If $\left\|g_{k}\right\|=0$, then terminate; else go to the next step.

Step 3. $\alpha_{k}$ is computed based on (24).

Step 4. Set $S_{k+1}=S_{k}+\alpha_{k} h_{k}^{\text {new }}$.

Step 5. $h_{k}^{\text {new }}$ is computed based on (26).

Step 6. Set $k=k+1$ and go to Step 2 .

Theorem 2. If the search direction $h_{k}^{\text {new }}$ is calculated by (26), and the step length $\alpha_{k}$ meets the standard Armijo search conditions, for all $k \geq 0$, and then one has

$$
g_{k}^{T} h_{k}^{\text {new }} \leq-\left(1-c_{1}\right)\left\|g_{k}\right\|^{2}
$$

Proof. When $k=0$, we can obtain

$$
g_{k}^{T} h_{k}^{\text {new }}=-\left\|g_{k}\right\|^{2}
$$

Then Theorem 2 is proved.

When $k \geq 1$, because the step length $\alpha_{k}$ is calculated by formula (24), $\alpha_{k} \leq 1$, and then we can obtain

$$
\begin{aligned}
g_{k}^{T} h_{k}^{\text {new }} & =-\left\|g_{k}\right\|^{2}+\beta_{k}^{\text {PRP }} \min \left\{\theta_{k-1}, 1\right\} g_{k}^{T} h_{k}^{\text {new }} \\
& \leq-\left\|g_{k}\right\|^{2}+\frac{\left\|g_{k}\right\|\left\|g_{k}-g_{k-1}\right\|}{\left\|g_{k-1}\right\|^{2}} \theta_{k-1}\left|g_{k}^{T} h_{k}^{\text {new }}\right| \\
& \leq-\left\|g_{k}\right\|^{2}+\frac{L_{k}\left\|v_{k-1}\right\|\left\|h_{k}^{\text {new }}\right\|}{\left\|g_{k-1}\right\|^{2}} \theta_{k-1}\left\|g_{k}\right\|^{2} \\
& \leq-\left\|g_{k}\right\|^{2}+\frac{L_{k}\left\|h_{k}^{\text {new }}\right\|^{2}}{\left\|g_{k-1}\right\|^{2}} \theta_{k-1}\left\|g_{k}\right\|^{2} \\
& =-\left(1-c_{1}\right)\left\|g_{k}\right\|^{2} .
\end{aligned}
$$

Hence, the conclusion is established.

3.5. Global Convergence. Under the following assumptions, the global convergence of the algorithm is established.

Assumption A. (i) The objective function is bounded in the following level:

$$
\Omega=\left\{s \in R^{n} \mid f(s) \leq f\left(s_{0}\right)\right\} .
$$

(ii) The gradient $g$ is Lipschitz continuous; that is, there exists a constant $L>0$ such that, for any $x, y \in \Omega$, we obtain

$$
\|g(x)-g(y)\| \leq L\|x-y\| .
$$


Lemma 3. Suppose that Assumption A holds, the step length $\alpha_{k}$ is calculated by (24) and the search direction $h_{k}^{\text {new }}$ is calculated by (26), and there is a constant $c_{2}>0$, then

$$
\begin{aligned}
\alpha_{k} & \geq \frac{\left(-g_{k}^{T} h_{k}^{\text {new }}\right)}{\left\|h_{k}^{\text {new }}\right\|^{2}}, \\
\sum_{k=0}^{\infty} \frac{\left\|g_{k}\right\|^{4}}{\left\|h_{k}^{\text {new }}\right\|^{2}} & <\infty .
\end{aligned}
$$

Proof. Set $c_{2}=\min \left\{\left(1-c_{1}\right), \rho(1-\delta) / L\right\}$, because the step length $\alpha_{k}$ is calculated by (24), then $\alpha_{k} \leq 1$.

When $\alpha_{k}=1$, according to Theorem 2, we can obtain the following:

$$
g_{k}^{T} h_{k}^{\text {new }} \leq-\left(1-c_{1}\right)\left\|g_{k}\right\|^{2} .
$$

Because $-g_{k}^{T} h_{k}^{\text {new }} \leq\left\|g_{k}\right\|\left\|h_{k}^{\text {new }}\right\|$, then we obtain

$$
\left\|h_{k}^{\text {new }}\right\| \geq\left(1-c_{1}\right)\left\|g_{k}\right\| \text {. }
$$

Then one has

$$
\left\|h_{k}^{\text {new }}\right\|^{2} \geq\left(1-c_{1}\right)\left\|g_{k}\right\|\left\|h_{k}^{\text {new }}\right\| \geq-\left(1-c_{1}\right) g_{k}^{T} h_{k}^{\text {new }} .
$$

We have

$$
\alpha_{k}=1 \geq \frac{-\left(1-c_{1}\right) g_{k}^{T} h_{k}^{\text {new }}}{\left\|h_{k}^{\text {new }}\right\|^{2}} .
$$

Hence, (33) is proved.

When $\alpha_{k}<1$, we know that $\rho^{-1} \alpha_{k}$ can not meet (24) according to the definition of the step length, and then

$$
f\left(s_{k}+\rho^{-1} \alpha_{k} h_{k}^{\text {new }}\right)>f\left(s_{k}\right)+\delta \rho^{-1} \alpha_{k} g_{k}^{T} h_{k}^{\text {new }} .
$$

According to the mean value theorem, we have

$$
\begin{aligned}
f & \left(s_{k}+\rho^{-1} \alpha_{k} h_{k}^{\text {new }}\right)-f\left(s_{k}\right) \\
& =\rho^{-1} \alpha_{k} g\left(s_{k}+t_{k} \rho^{-1} \alpha_{k} h_{k}^{\text {new }}\right)^{2} h_{k}^{\text {new }} \\
& \leq \rho^{-1} \alpha_{k} g_{k}^{T} h_{k}^{\text {new }}+L \rho^{-2} \alpha_{k}^{2}\left\|h_{k}^{\text {new }}\right\|^{2},
\end{aligned}
$$

where $t_{k} \in(0,1)$.

According to the formula, we obtain

$$
\delta \rho^{-1} \alpha_{k} g_{k}^{T} h_{k}^{\text {new }} \leq \rho^{-1} \alpha_{k} g_{k}^{T} h_{k}^{\text {new }}+L \rho^{-2} \alpha_{k}^{2}\left\|h_{k}^{\text {new }}\right\|^{2} .
$$

So we have

$$
\alpha_{k} \geq-\frac{\rho(1-\delta) g_{k}^{T} h_{k}^{\text {new }}}{L\left\|h_{k}^{\text {new }}\right\|^{2}} \geq c_{2} \frac{\left(g_{k}^{T} h_{k}^{\text {new }}\right)}{\left\|h_{k}^{\text {new }}\right\|^{2}} .
$$

Hence, (33) is proved.

We can see that $\left\{f_{k}\right\}$ is monotone bounded according to (24) and Assumption A, so we can obtain the following conclusion based on (24) and (33) and Lemma 3:

$$
\begin{gathered}
\sum_{k=0}^{k} \delta c_{2}\left(1-c_{1}\right)^{2} \frac{\left\|g_{k}\right\|^{4}}{\left\|h_{k}^{\text {new }}\right\|^{2}} \leq \sum_{k=0}^{k} \delta c_{2} \frac{\left(-g_{k}^{T} h_{k}^{\text {new }}\right)^{2}}{\left\|h_{k}^{\text {new }}\right\|^{2}} \\
\leq \sum_{k=0}^{k}-\delta \alpha_{k} g_{k}^{T} h_{k}^{\text {new }} \leq \sum_{k=0}^{k}\left(f_{k}-f_{k+1}\right)<\infty .
\end{gathered}
$$

Hence, (34) is proved.
Theorem 4. Suppose that the step length $\alpha_{k}$ and the search direction $h_{k}^{\text {new }}$ are generated by IME-NCG algorithm, and then one has that

$$
\lim _{k \rightarrow \infty}\left\|g_{k}\right\|=0
$$

Proof. Owing to the fact that the step length $\alpha_{k}$ and the search direction $h_{k}^{\text {new }}$ are generated by (24) and (26), respectively, then

$$
\begin{aligned}
& \left\|h_{k}^{\text {new }}\right\| \leq\left\|g_{k}\right\|+\left|\beta_{k}\right| \min \left\{\theta_{k-1}, 1\right\}\left\|h_{k-1}^{\text {new }}\right\| \\
& \leq\left\|g_{k}\right\|+\frac{\left\|g_{k}\right\|\left\|g_{k}-g_{k-1}\right\|}{\left\|g_{k-1}\right\|^{2}} \frac{\left\|c_{1}\right\|\left\|g_{k-1}\right\|^{2}}{\left\|h_{k}^{\text {new }}\right\|^{2} L_{k}}\left\|h_{k}^{\text {new }}\right\| \\
& \leq\left\|g_{k}\right\| \\
& \quad+\frac{\left\|g_{k}\right\|\left\|g_{k}-g_{k-1}\right\|}{\left\|g_{k-1}\right\|^{2}} \frac{\left\|c_{1}\right\|\left\|g_{k-1}\right\|^{2}\left\|s_{k-1}\right\|}{\left\|h_{k}^{\text {new }}\right\|^{2}\left\|g_{k}-g_{k-1}\right\|}\left\|h_{k}^{\text {new }}\right\| \\
& =\left\|g_{k}\right\|+c_{1} \alpha_{k}\left\|g_{k}\right\| \leq\left(1+c_{1}\right)\left\|g_{k}\right\| .
\end{aligned}
$$

So we can obtain

$$
\frac{\left\|g_{k}\right\|^{4}}{\left\|d_{k}\right\|^{2}} \geq \frac{\left\|g_{k}\right\|^{4}}{\left\|d_{k}\right\|^{2}\left(1+c_{1}\right)^{2}}=\frac{\left\|g_{k}\right\|^{2}}{\left(1+c_{1}\right)^{2}} .
$$

Hence, (34) is proved.

\section{The Engineering Application}

4.1. Experimental Set-Up. In this paper, a diagram of experimental set-up is organized in Figure 2. The size of the coal-seam structure specimen manufactured artificially was $2000 \mathrm{~mm} \times 800 \mathrm{~mm} \times 1750 \mathrm{~mm}$, the pick is mounted on the rotary cutting arm, it can be driven by the reducer and torque, and its power driven by the motor is rated at $55 \mathrm{~kW}$ [48]. The cutting bench achieves free forward and backward movement by the hydraulic pressure drive control system. System output responses can be measured by the corresponding sensors, then converted by a signal amplifier, and finally recorded by V10Dasp data vibration signal acquisition system $[49,50]$.

4.2. The Engineering Application. All codes were written in MATLAB 7.0 and run on a HP with 2.0 GB RAM and Windows 7 operating system. Stop the iteration if criterion is defined as $\left|g_{k}\right| \leq 10^{-5}$ being satisfied or run time more than 500 seconds. And the step length $\alpha_{k}$ is calculated by (24), and the parameters are obtained as follows: $\delta=$ $0.45, \rho=0.12, L_{0}=10^{-4}, c_{1}=1-10^{-6}, p_{i}=1.3$, $i=1,2, \ldots, n$, and $\xi=2 \times 10^{-10}$. For performance analysis of dynamic force identification techniques used, the performance measurement metrics for dynamic force are described as follows: restoration time, iterative steps, and root mean-square-error (RMSE), where RMSE is defined by

$$
\text { RMSE }=\sqrt{\frac{1}{N} \sum_{i=1}^{N}\left[S_{\text {id }}-S_{\text {actual }}\right]^{2}},
$$




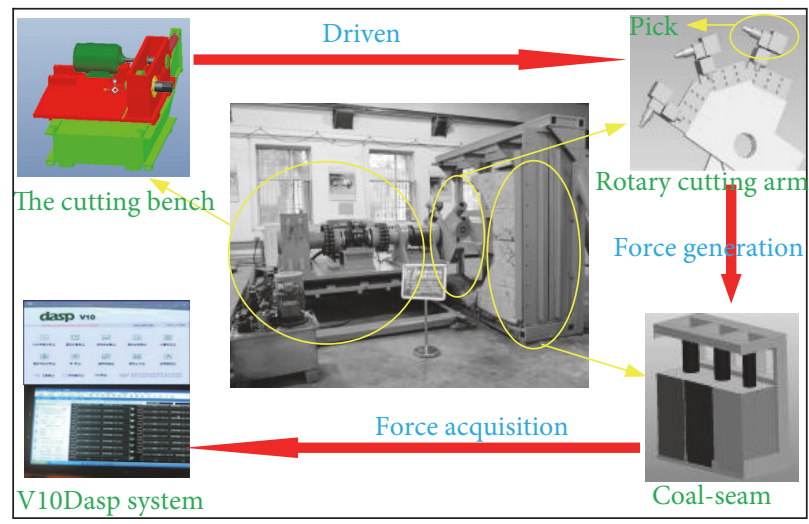

FIgURE 2: A diagram of experimental set-up.

where $S_{\text {actual }}$ is the actual measured random dynamic force and $S_{\text {id }}$ is the identified random dynamic force.

In this section, the material parameters of experimental conditions are shown in Tables 1 and 2.

It is generally known that direct numerical treatment fails to solve the present inverse problem in the engineering application. Therefore, the proposed method is to be applied to identify dynamic force. However, the key problem is how to select the suitable regularization parameter to obtain the optimal solution. Moreover, the suitable regularization parameter obtained by L-curve is really important for numerical experiment process.

First, the graphical illustration of L-curve was shown in Figure 3(a) according to L-curve criterion. Let it be noted that it is very possible to obtain the optimal solution by combining the L-curve criterion with the proposed regularization method.

It is clearly seen from Figure 3(a) that when the regularization parameter is small, the graph shows very clearly that the norm of regularized solution $\|S\|_{2}$ is large, but the norm of the corresponding residual $\left\|G S-Y^{c}\right\|_{2}$ is small; we can speculate that it is likely to be caused by measurement errors. However, when the regularization parameter is large, it is found that the norm of regularized solution $\|S\|_{2}$ is a poor approximation; on the contrary, the norm of the corresponding residual $\left\|G S-Y^{c}\right\|_{2}$ is large.

The corner of the L-curve indicates this transition, owing to the fact it shows a compromise between the minimization of the norm of the residual $\left\|G S-Y^{c}\right\|_{2}$ and norm of the solution $\|S\|_{2}$. The selection of the regularization parameter of IME regularization can be not completely guaranteed to be suitable for all systems with the ill-posedness. However, many examples show that the L-curve criterion is a powerful method for determining a suitable value of the regularization parameter for many significant engineering and mathematical problems.

In order to further discuss the influence of L-curve on numerical experiments, then make sure that the optimal regularization parameter is selected from the L-curve.
According to the L-curve criterion, the value of different regularization parameters are selected from Figure 3(a) to find the optimal regularization parameter. The measured displacement response was shown in Figure 4. And the identified results were formed in Figures 3(b), 3(c), 3(d), and 3(e).

Figures 3(d) and 3(e) show that the identified result is extremely unsatisfactory due to small regularization parameter. However, we can see from Figure 3(b) that even if when the regularization parameter is large, that is, beyond a certain threshold, the identified result is far away from what is normal. At the same time, the identified result of the value at the corner of the L-curve is shown in Figure 3(c). Figure 3(c) clearly shows that the most accurate results are obtained under the optimal regularization parameter $(\lambda=$ $10^{-2}$ ) compared with other cases of regularization parameters. So we conclude that the values at the corner of the L-curve are selected as the most suitable regularization parameter.

As we can see from the above discussion, the regularization parameter was obtained by L-curve method, and $\lambda_{\text {L-curve }}=0.01$. Then identified dynamic force can be given based on measured displacement response using the identified modal model and the proposed algorithm, and the identified results were formed in Figure 5 by assembling the analysis results of ME, ME-CG, ME-NCG, IME-CG, and IME-NCG algorithm.

It was obtained that the random dynamic force is easily identified with application of the above identification algorithms, and the identified random dynamic force is obviously observed using the regularization parameter selected by Lcurve method as seen in Figures 5(a), 5(b), 5(c), 5(d), and 5(e), respectively.

Figure 5(a) shows that the identified result is obtained using ME algorithm, Figure 5(b) shows that the identified result is obtained using $\mathrm{ME}-\mathrm{CG}$ algorithm, identified result using ME-NCG algorithm is obtained from Figure 5(c), Figure $5(\mathrm{~d})$ shows that the identified result is obtained using IME-CG algorithm, and the identified result using IME-NCG algorithm is shown in Figure 5(e). 


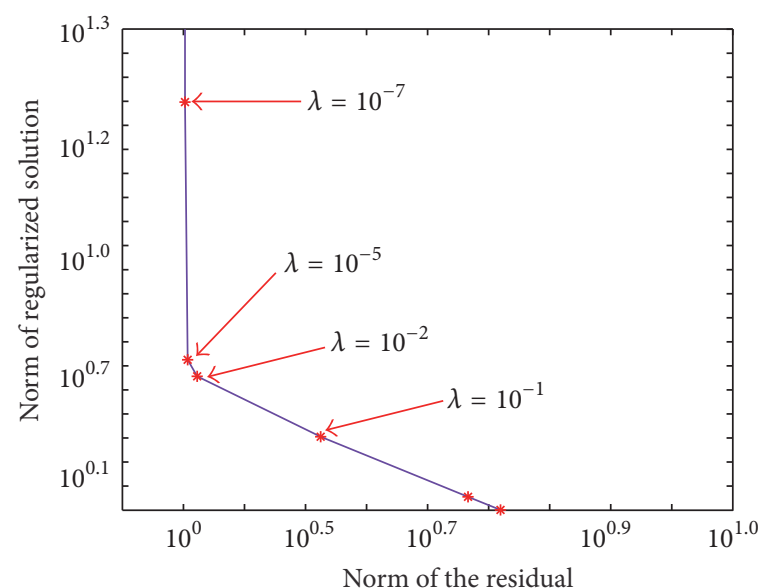

(a) The graphical illustration of L-curve

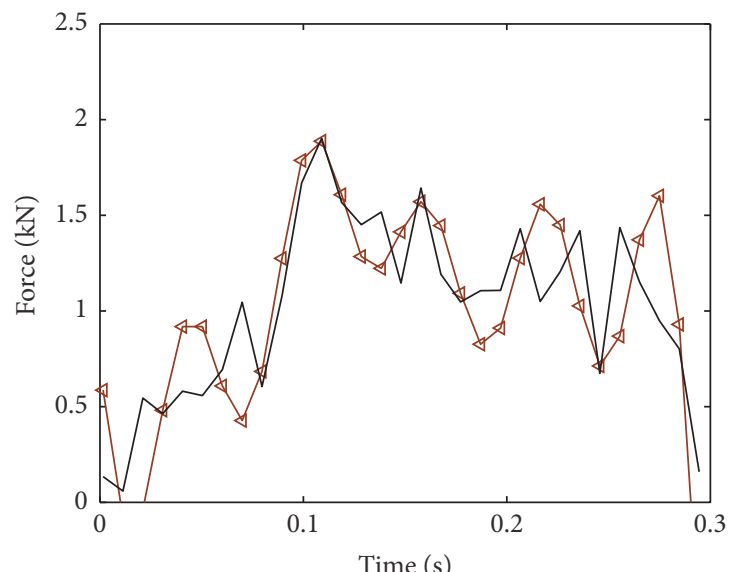

The identified force

The actual force

(c) $\lambda=10^{-2}$

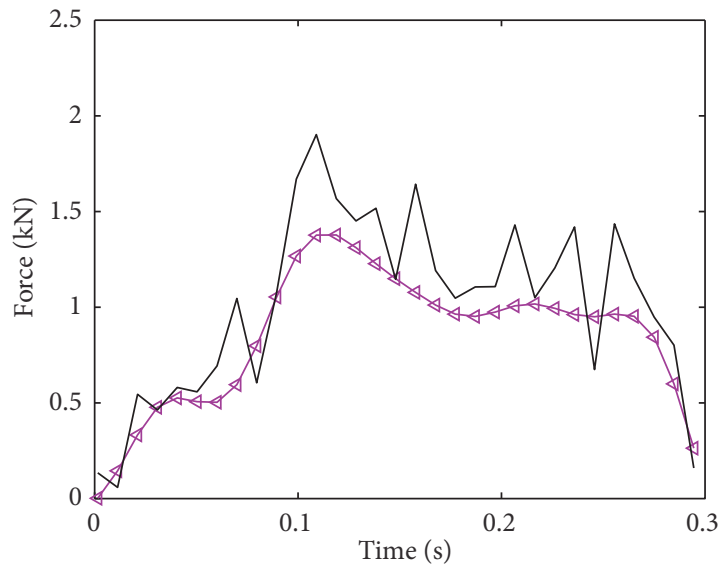

$\varangle$ The identified force

The actual force

(b) $\lambda=10^{-1}$

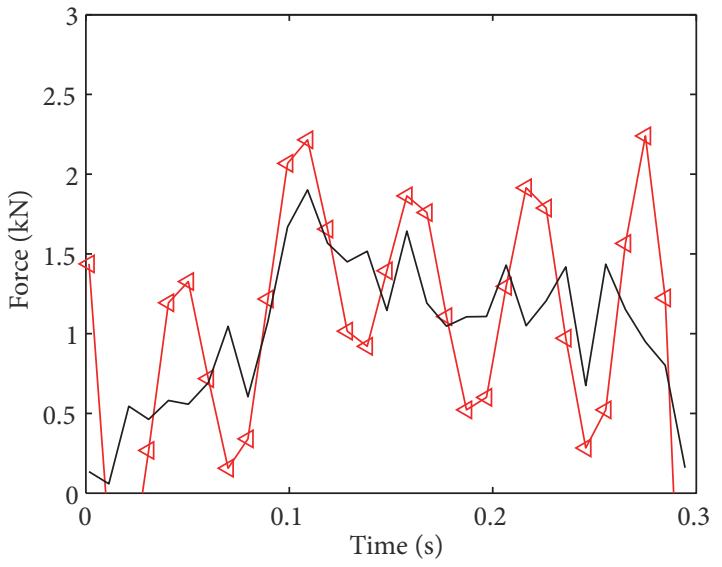

$\dashv$ The identified force

- The actual force

(d) $\lambda=10^{-5}$

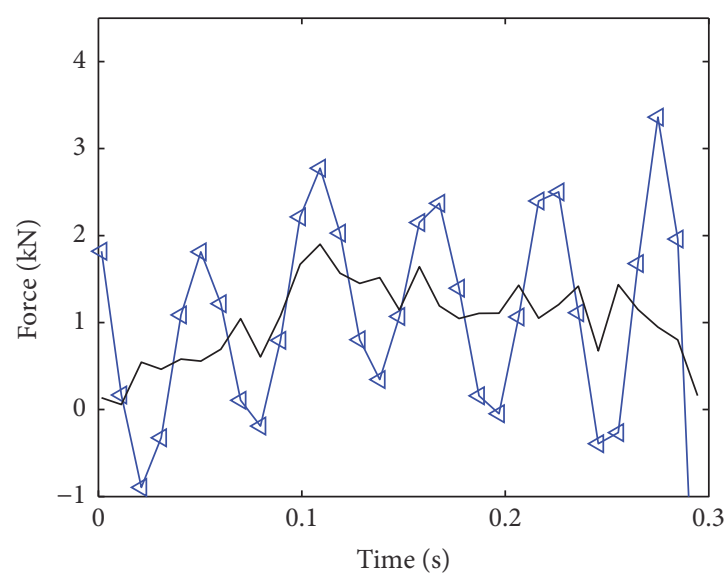

$\triangleleft$ The identified force

- The actual force

(e) $\lambda=10^{-7}$

FIgURE 3: The selection of the suitable regularization parameter from the L-curve. 


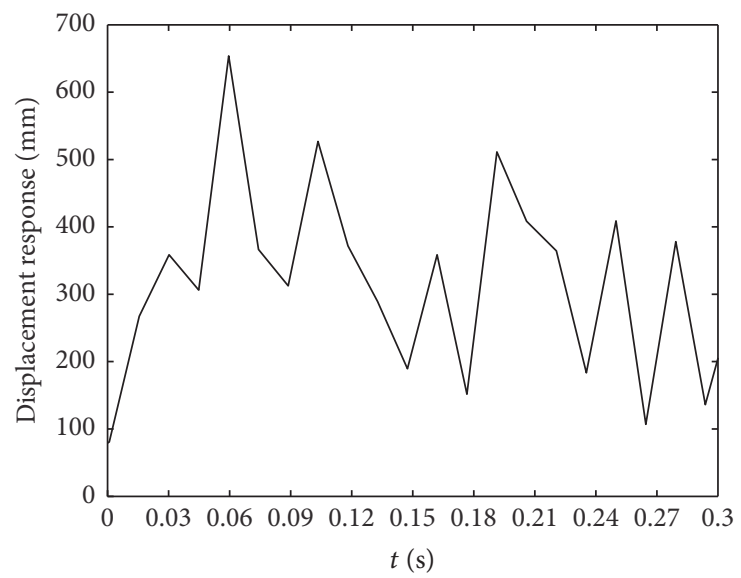

FIGURE 4: Measured displacement response.

Moreover, it can be shown that different identification algorithms can commendably identify the random dynamic force. However, to some extent, the performance of random dynamic force identification has a certain difference.

We analyzed the different identification algorithms at $\lambda_{\text {L-curve }}=0.01$. And restoration time, iterative steps, and RMSE were used to evaluate the quality of dynamic force identification.

In Table 3, it was found that the proposed IME-NCG algorithm is associated with minimum RMSE, minimum restoration time, and minimum iterative steps in comparison to ME, ME-CG, ME-NCG, and IME-CG algorithm, which proves that the IME-NCG algorithm is superior to the other algorithms in the identification of random dynamic force at $\lambda_{\mathrm{L} \text {-curve }}=0.01$, which is closer to the actual dynamic force.

However, as is well known, different regularization parameters are obtained by different regularization parameter selection methods, which will lead to different identified results. In this paper, the GCV is applied to compute the regularization parameter compared with L-curve method.

Figure 6 shows that the regularization parameter was obtained by GCV method, when $\lambda_{\mathrm{GCV}}=0.006, \mathrm{GCV}(\lambda)$ reaches the minimum, and the identified results were formed in Figure 7 by assembling the analysis results of different algorithms at $\lambda_{\mathrm{GCV}}=0.006$.

In Figures 7(a), 7(b), 7(c), 7(d), and 7(e), it can be shown that five different identification algorithms can identify the random dynamic force, and it was found that the proposed IME-NCG algorithm is associated with minimum RMSE, minimum restoration time, and minimum iterative steps in comparison to the other algorithm from Table 4; hence, we can draw a conclusion that the IME-NCG algorithm is closer to the actual force at $\lambda_{\mathrm{GCV}}=0.006$, which shows that the IME-NCG algorithm is superior to the other algorithms whether the regularization parameter is selected by using Lcurve or GCV method.
From Tables 3 and 4, we can see that RMSE, restoration time, and iteration steps using L-curve method are lower than those of using GCV method whether using IME-NCG algorithm or the other algorithms.

Hence, we can conclude that the IME-NCG algorithm combined with L-curve method is available to identify the dynamic force.

\section{Conclusions}

In this paper, the identification model for random dynamic force between pick and coal-seam is established through the relationship between the identified force vector and the displacement responses measured by machinery vibration system. Then the entropy item of maximum entropy (ME) regularization is redesigned using the 1-norm estimation method and the elongated entropy function according to the ill-conditioned characteristics of force identification, which was named as an improved maximum entropy (IME) regularization technique, was proposed to process the random dynamic force. Then the force identification problem is transformed into an unconstrained optimization problem and a novel conjugate gradient (NCG) method was applied to solve the objective function, which was called IME-NCG algorithm.

The results show that the proposed IME-NCG algorithm is associated with minimum RMSE, minimum restoration time, and minimum iterative steps in comparison to $\mathrm{ME}$, ME-CG, ME-NCG, and IME-CG algorithm, and L-curve method is introduced which is better than GCV method and is applied to select the regularization parameter. Thus we can safely conclude that the combination of IME-NCG algorithm and L-curve method can reduce the ill-posedness of random dynamic force identification and achieve the optimal solution of inverse problem in practical engineering. 

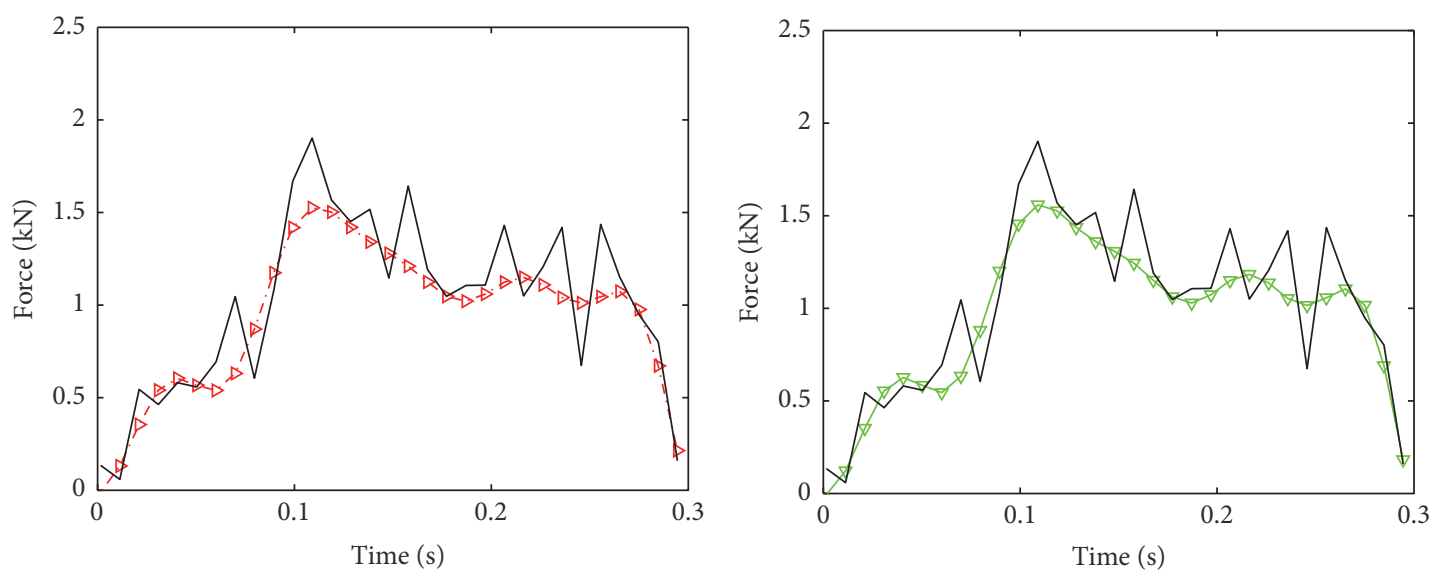

$\rightarrow-$ The identified force

_ The actual force

(a) ME algorithm

$\nabla$ The identified force

- The actual force

(b) ME-CG algorithm
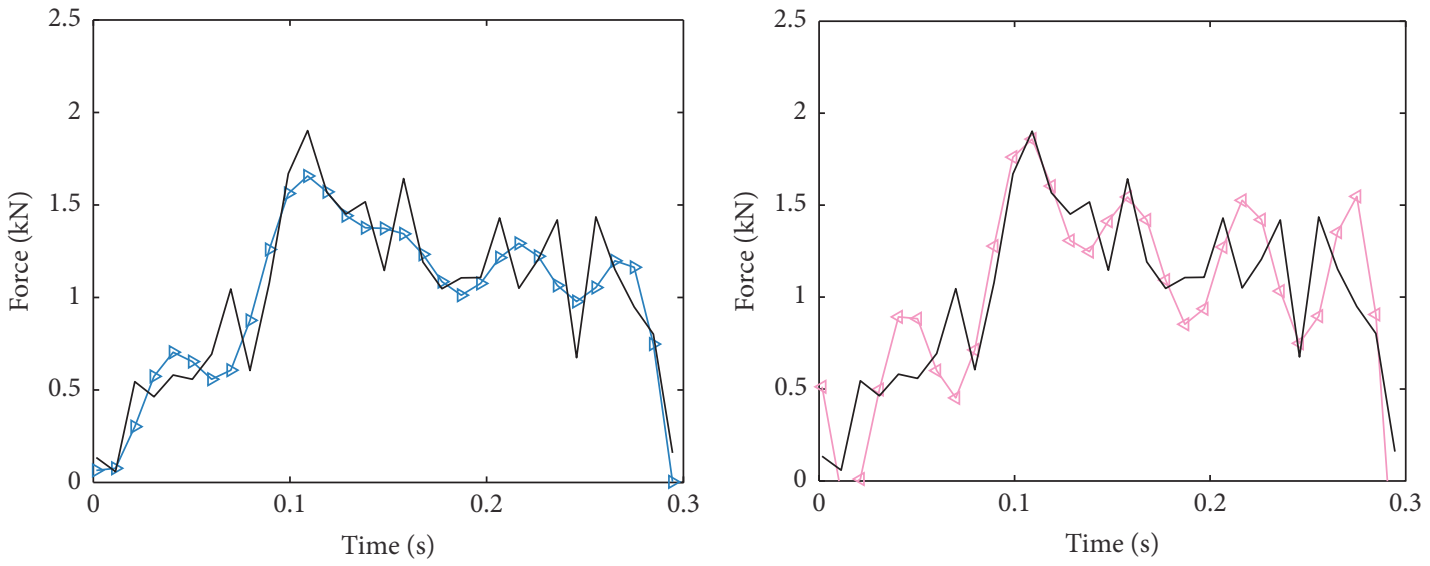

$\rightarrow$ The identified force

The actual force

4- The identified force

- The actual force

(c) ME-NCG algorithm

(d) IME-CG algorithm

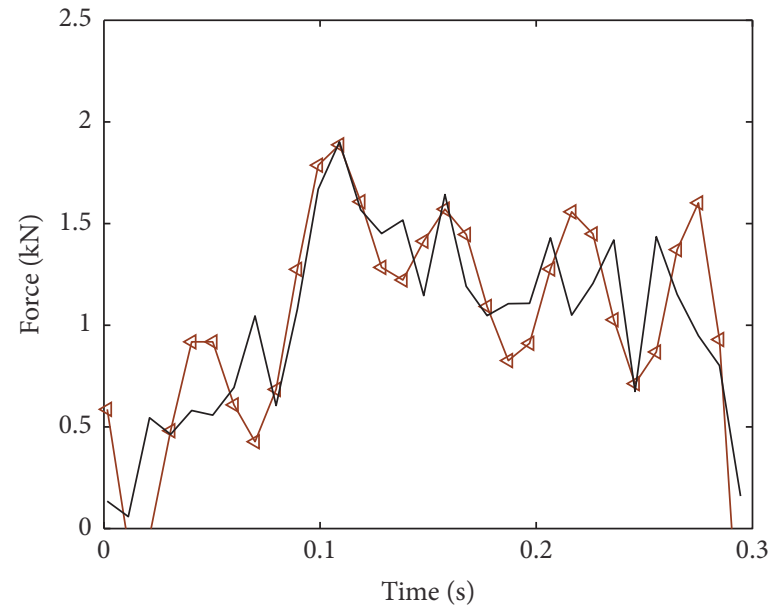

$\varangle$ The identified force

- The actual force

(e) IME-NCG algorithm

FIGURE 5: Identified results of different algorithms at $\lambda_{\text {L-curve }}=0.01$. 


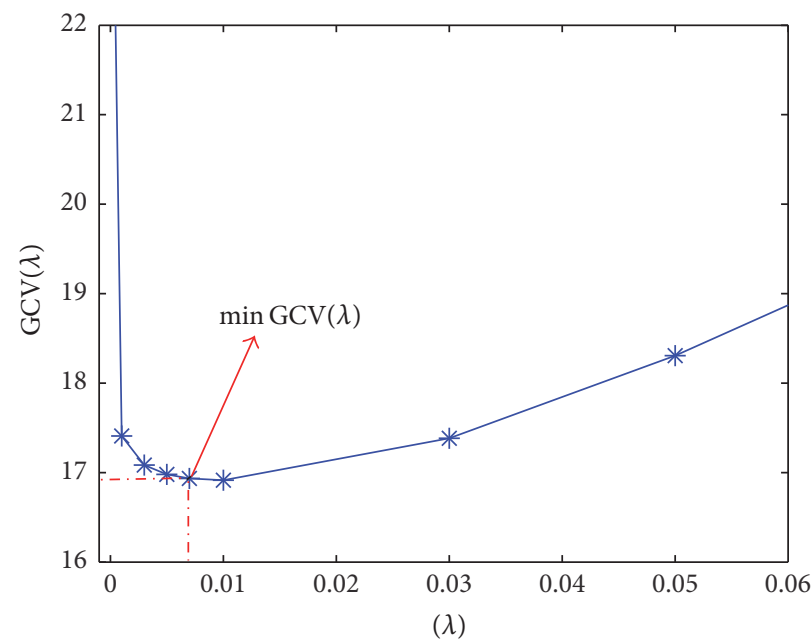

FIGURE 6: Selection of regularization parameters using GCV method.

TABLE 1: The material parameters.

\begin{tabular}{lcccc}
\hline Materials & $\begin{array}{c}\text { Compressive } \\
\text { strength } \\
(\mathrm{MPa})\end{array}$ & $\begin{array}{c}\text { Elastic } \\
\text { modulus } \\
(\mathrm{MPa})\end{array}$ & $\begin{array}{c}\text { Poisson } \\
\text { ratio }\end{array}$ & $\begin{array}{c}\text { Density } \\
\left(\mathrm{kg} / \mathrm{m}^{3}\right)\end{array}$ \\
\hline Coal-seam & 2.68 & 322.09 & 0.25 & 1607.98 \\
Pick & - & 600 & 0.25 & 7800 \\
\hline
\end{tabular}

TABLE 2: The experimental conditions.

\begin{tabular}{lccc}
\hline $\begin{array}{l}\text { Installation } \\
\text { angle }\left({ }^{\circ}\right)\end{array}$ & $\begin{array}{c}\text { Maximum cutting } \\
\text { thickness }(\mathrm{mm})\end{array}$ & $\begin{array}{c}\text { Hauling } \\
\text { speed }(\mathrm{r} / \mathrm{min})\end{array}$ & $\begin{array}{c}\text { Rotary arm } \\
\text { speed }(\mathrm{m} / \mathrm{min})\end{array}$ \\
\hline 35 & 15 & 0.6 & 25 \\
\hline
\end{tabular}

TABLE 3: The measurement metrics comparisons using L-curve method.

\begin{tabular}{lccccc}
\hline Method & ME algorithm & ME-CG algorithm & ME-NCG algorithm & IME-CG algorithm & ME-NCG algorithm \\
\hline Restoration time (s) & 36.0152 & 25.4632 & 18.6788 & 10.1442 & 3.0421 \\
Iterative steps & 30 & 25 & 19 & 15 & 8 \\
RMSE & 0.4702 & 0.3775 & 0.1867 & 0.0828 & 0.0783 \\
\hline
\end{tabular}

TABLE 4: The measurement metrics comparisons using GCV method.

\begin{tabular}{lccccc}
\hline Method & ME algorithm & ME-CG algorithm & ME-NCG algorithm & IME-CG algorithm & ME-NCG algorithm \\
\hline Restoration time (s) & 40.1442 & 28.3664 & 20.4112 & 17.1100 & 5.0021 \\
Iterative steps & 35 & 28 & 22 & 18 & 10 \\
RMSE & 0.6047 & 0.4241 & 0.2827 & 0.0919 & 0.0806 \\
\hline
\end{tabular}




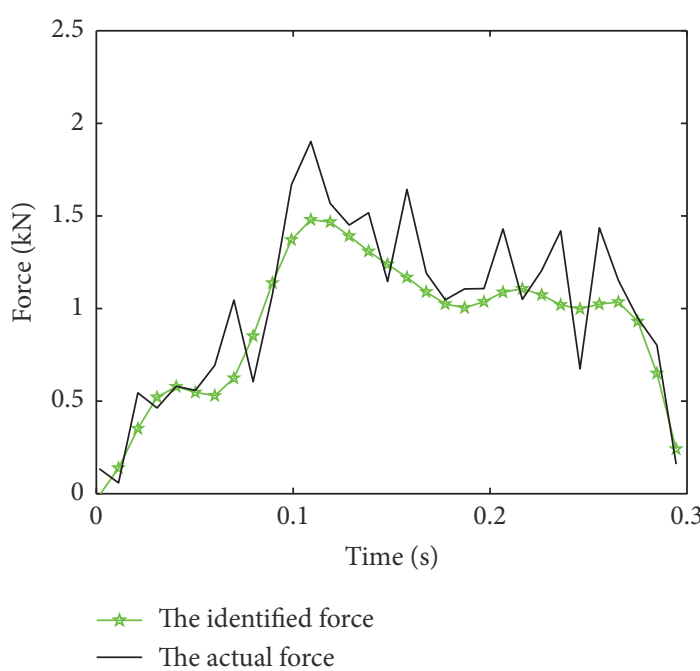

(a) ME algorithm

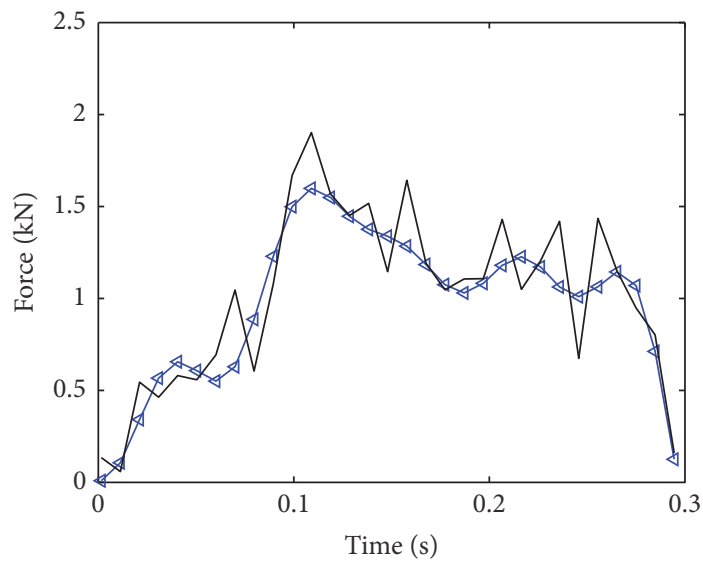

$\varangle$ The identified force

- The actual force

(c) ME-NCG algorithm

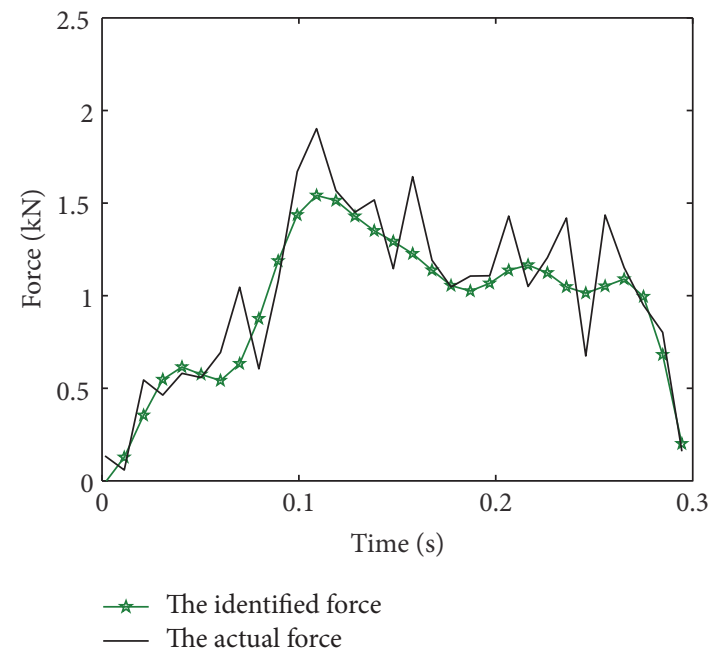

(b) ME-CG algorithm

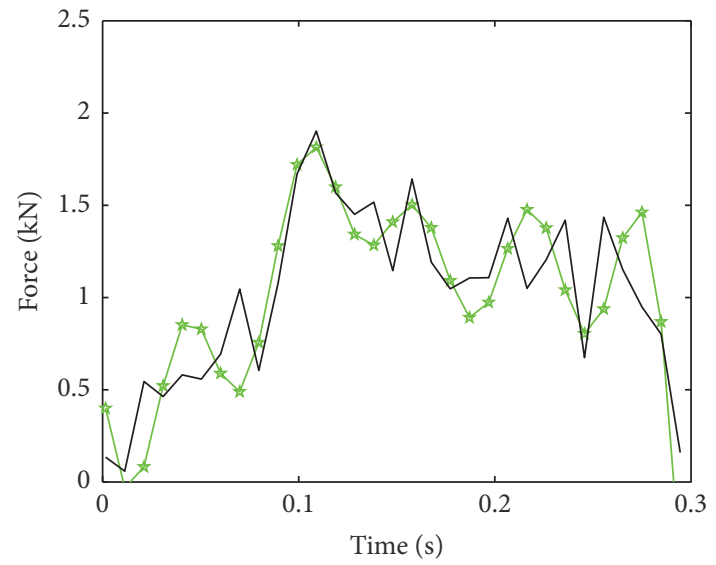

$\therefore$ The identified force

- The actual force

(d) IME-CG algorithm

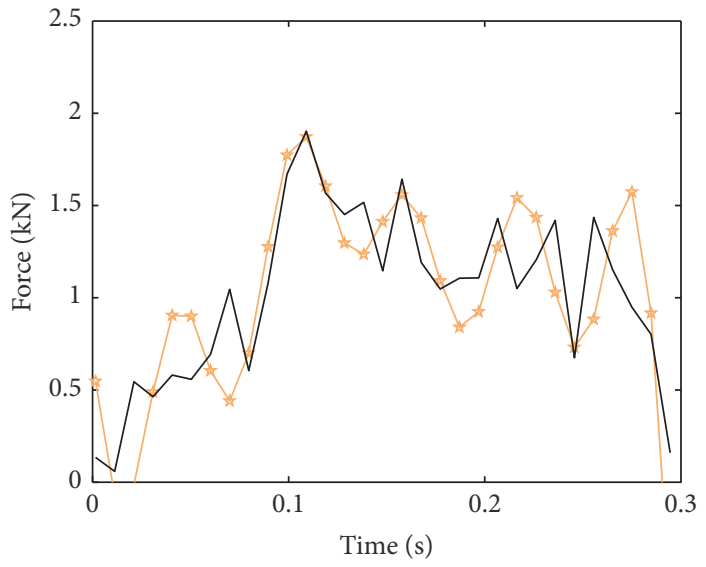

$x$ The identified force

- The actual force

(e) IME-NCG algorithm

FIGURE 7: Identified results of different algorithms at $\lambda_{\mathrm{GCV}}=0.006$. 


\section{Conflicts of Interest}

The authors declare that they have no conflicts of interest.

\section{Acknowledgments}

This work was supported by the Chinese National Natural Science Foundation (Contract nos. 51674106 and 51274091).

\section{References}

[1] T. Poggio, V. Torre, and C. Koch, "Computational vision and regularization theory," Nature, vol. 317, no. 6035, pp. 314-319, 1985.

[2] T. S. Jang, H. G. Sung, S. L. Han, and S. H. Kwon, "Inverse determination of the loading source of the infinite beam on elastic foundation," Journal of Mechanical Science \& Technology, vol. 22, no. 12, pp. 2350-2356, 2008.

[3] J. M. Varah, "A practical examination of some numerical methods for linear discrete ill-posed problems," SIAM Review, vol. 21, no. 1, pp. 100-111, 1979.

[4] Y. Dai, L. Liu, and S. Feng, "On the identification of coupled pitch and heave motions using opposition-based particle swarm optimization," Mathematical Problems in Engineering, vol. 2014, Article ID 784049, 10 pages, 2014.

[5] W. Luo, "Parameter identifiability of ship manoeuvring modeling using system identification," Mathematical Problems in Engineering, vol. 2016, Article ID 8909170, 10 pages, 2016.

[6] L. Shi, H. He, and W. Yan, "Prestress force identification for externally prestressed concrete beam based on frequency equation and measured frequencies," Mathematical Problems in Engineering, vol. 2014, Article ID 840937, 13 pages, 2014.

[7] J. Gao, D. Wang, and J. Peng, "A Tikhonov-type regularization method for identifying the unknown source in the modified Helmholtz equation," Mathematical Problems in Engineering, vol. 2012, Article ID 878109, 13 pages, 2012.

[8] X. Liu, S. Liu, and H. Ji, "Mechanism of rock breaking by pick assisted with water jet of different modes," Journal of Mechanical Science and Technology, vol. 29, no. 12, pp. 5359-5368, 2015.

[9] J. M. Varah, "On the numerical solution of ill-conditioned linear systems with applications to ill-posed problems," SIAM Journal on Numerical Analysis, vol. 10, pp. 257-267, 1973.

[10] K. M. Hanke and L. P. C. Hansen, "Regularization methods for large-scale problems," Surveys on Mathematics for Industry, vol. 3, no. 4, pp. 253-315, 1993.

[11] E. Talic, A. Schirrer, M. Kozek, and S. Jakubek, "Multi-objective parameter identification of Euler-Bernoulli beams under axial load," Journal of Sound and Vibration, vol. 341, pp. 86-99, 2015.

[12] T. S. Jang, H. Baek, S. L. Hana, and T. Kinoshita, "Indirect measurement of the impulsive load to a nonlinear system from dynamic responses: inverse problem formulation," Mechanical Systems and Signal Processing, vol. 24, no. 6, pp. 1665-1681, 2010.

[13] Y. Jia, Z. Yang, N. Guo, and L. Wang, "Random dynamic load identification based on error analysis and weighted total least squares method," Journal of Sound and Vibration, vol. 358, pp. 111-123, 2015.

[14] S. J. Phillips and R. E. Schapire, "Maximum entropy density estimation with generalized regularization and an application to species distribution modeling," Journal of Machine Learning Research (JMLR), vol. 8, no. 2, pp. 1217-1260, 2007.
[15] U. Amato and W. Hughes, "Maximum entropy regularization of Fredholm integral equations of the first kind," Inverse Problems, vol. 7, no. 6, 793 pages, 1991.

[16] Y.-W. Chiang, P. P. Borbat, and J. H. Freed, "Maximum entropy: A complement to Tikhonov regularization for determination of pair distance distributions by pulsed ESR," Journal of Magnetic Resonance, vol. 177, no. 2, pp. 184-196, 2005.

[17] A. Mohammad-Djafari, J.-F. Giovannelli, G. Demoment, and J. Idier, "Regularization, maximum entropy and probabilistic methods in mass spectrometry data processing problems," International Journal of Mass Spectrometry, vol. 215, no. 1-3, pp. 175-193, 2002.

[18] P. Haffner, S. Phillips, and R. Schapire, "Efficient multiclass implementations of L1-regularized maximum entropy," Computer Science, abs/cs/0506101, 2005.

[19] T. W. Grandy, "Maximum-entropy and Bayesian methods in inverse problems," Acta Applicandae Mathematica, vol. 20, no. 1-2, pp. 189-191, 1990.

[20] J. Myrheim and H. Rue, "New algorithms for maximum entropy image restoration," Graphical Models \& Image Processing, vol. 54, no. 3, pp. 223-238, 1992.

[21] W. Zhu and T. Yan, "The conjugate gradient algorithm for solving the absolute value equations based on maximum entropy function," Journal of Hefei Normal University, 2016.

[22] G. Nicolas, J. Andrew, and C. C. Finlay, "Maximum entropy regularization of time-dependent geomagnetic field models," Geophysical Journal of the Royal Astronomical Society, vol. 171, no. 3, pp. 1005-1016, 2010.

[23] J. Liu, X. Sun, X. Han, C. Jiang, and D. Yu, "Dynamic load identification for stochastic structures based on Gegenbauer polynomial approximation and regularization method," Mechanical Systems and Signal Processing, vol. 56-57, pp. 35-54, 2015.

[24] J. Liu, X. Sun, X. Meng et al., "A novel shape function approach of dynamic load identification for the structures with interval uncertainty," International Journal of Mechanics \& Materials in Design, no. 3, pp. 1-2, 2015.

[25] L. Wang, X. Han, J. Liu, X. He, and F. Huang, "A new regularization method and application to dynamic load identification problems," Inverse Problems in Science and Engineering, vol. 19, no. 6, pp. 765-776, 2011.

[26] W. Chen, "A new extrapolation algorithm for band-limited signals using the regularization method," IEEE Transactions on Signal Processing, vol. 41, no. 3, pp. 1048-1060, 1993.

[27] V. Melicher and V. Vrabel, "On a continuation approach in Tikhonov regularization and its application in piecewiseconstant parameter identification," Inverse Problems, vol. 29, no. 11, pp. 193-212, 2013.

[28] Z. Zhao and Z. Meng, "A modified Tikhonov regularization method for a backward heat equation," Inverse Problems in Science and Engineering, vol. 19, no. 8, pp. 1175-1182, 2011.

[29] H. Cheng, X.-L. Feng, and C.-L. Fu, "A mollification regularization method for the Cauchy problem of an elliptic equation in a multi-dimensional case," Inverse Problems in Science and Engineering, vol. 18, no. 7, pp. 971-982, 2010.

[30] A. Qian and Y. Gui, "Determining an unknown source in the heat equation by a mollification regularization method," in Proceedings of the 2010 International Conference on Computational Intelligence and Software Engineering (CiSE '10), pp. 1-4, IEEE, Wuhan, China, December 2010. 
[31] F. Yang, C.-L. Fu, and X.-X. Li, "A mollification regularization method for identifying the time-dependent heat source problem," Journal of Engineering Mathematics, vol. 522, no. 18, pp. 1-14, 2016.

[32] S. Marchesiello and L. Garibaldi, "A time domain approach for identifying nonlinear vibrating structures by subspace methods," Mechanical Systems and Signal Processing, vol. 22, no. 1, pp. 81-101, 2008.

[33] E. Lourens, E. Reynders, G. De Roeck, G. Degrande, and G. Lombaert, "An augmented Kalman filter for force identification in structural dynamics," Mechanical Systems and Signal Processing, vol. 27, no. 1, pp. 446-460, 2012.

[34] M. S. Allen and T. G. Carne, "Delayed, multi-step inverse structural filter for robust force identification," Mechanical Systems and Signal Processing, vol. 22, no. 5, pp. 1036-1054, 2008.

[35] T. Reginska, "A regularization parameter in discrete ill-posed problems," Society for Industrial and Applied Mathematics, 1996.

[36] B. Movahedian and B. Boroomand, "Inverse identification of time-harmonic loads acting on thin plates using approximated Green's functions," Inverse Problems in Science and Engineering, vol. 24, no. 8, pp. 1475-1493, 2016.

[37] H. Du and M. Cui, "Approximate solution of the Fredholm integral equation of the first kind in a reproducing kernel Hilbert space," in Proceedings of the International Conference on Mathematics and Engineering Techniques in Medicine and Biological Scienes (METMBS '04), DBLP, pp. 322-328, Las Vegas, Nevada, USA, June 21-24, 2004.

[38] C. Xiang, Y. Tong, Z. Wu et al., "A new method for tsvd regularization truncated parameter selection," Mathematical Problems in Engineering, vol. 2013, Article ID 161834, 9 pages, 2013.

[39] H. Gzyl, "Ill-posed linear inverse problems and maximum entropy in the mean," Acta Científica Venezolana, vol. 53, no. 2, pp. 74-93, 2002.

[40] J.-M. Loubes and B. Pelletier, "Maximum entropy solution to illposed inverse problems with approximately known operator," Journal of Mathematical Analysis and Applications, vol. 344, no. 1, pp. 260-273, 2008.

[41] L. Jing, S. Liu, L. Zhihong, and S. Meng, "An image reconstruction algorithm based on the extended Tikhonov regularization method for electrical capacitance tomography," Measurement, vol. 42, no. 3, pp. 368-376, 2009.

[42] M. E. Hochstenbach, L. Reichel, and G. Rodriguez, "Regularization parameter determination for discrete ill-posed problems," Journal of Computational and Applied Mathematics, vol. 273, pp. 132-149, 2015.

[43] P. C. Hansen and D. P. O'Leary, "The use of the L-curve in the regularization of discrete ill-posed problems," SIAM Journal on Scientific Computing, vol. 14, no. 6, pp. 1487-1503, 1993.

[44] G. H. Golub, M. Heath, and G. Wahba, "Generalized crossvalidation as method for choosing a good ridge parameter," Technometrics. A Journal of Statistics for the Physical, Chemical and Engineering Sciences, vol. 21, no. 2, pp. 215-223, 1979.

[45] X.-P. Wu, L.-Y. Liu, F.-J. Xie, and Y.-F. Li, "A new conjugate gradient algorithm with sufficient descent property for unconstrained optimization," Mathematical Problems in Engineering, vol. 2015, Article ID 352524, 8 pages, 2015.

[46] L. Zhang, W. Zhou, and D.-H. Li, "A descent modified PolakRibière-Polyak conjugate gradient method and its global convergence," Ima Journal of Numerical Analysis, vol. 26, no. 4, pp. 629-640, 2006.
[47] C. S. Liu, "Novel algorithms based on the conjugate gradient method for inverting ill-conditioned matrices, and a new regularization method to solve ill-posed linear systems," Computer Modeling in Engineering and Sciences, vol. 60, no. 3, pp. 279-308, 2010.

[48] C. P. Ren and C. S. Liu, "Experimental research on properties of analog materials of coal and rock," Journal of Heilongjiang University of Science and Technology, vol. 24, no. 6, pp. 581-584, 2014.

[49] C.-S. Liu, C.-P. Ren, and D.-G. Li, "Reconstruction and deduction of cutting coal and rock load spectrum on modified discrete regularization algorithm," Journal of China Coal Society, vol. 39, no. 5, pp. 981-986, 2014.

[50] C. S. Liu, C. P. Ren, and F. Han, "Study on time-frequency spectrum characteristic of dynamic cutting load based on wavelet regularization," Applied Mechanics and Materials, vol. 577, pp. 196-200, 2014. 


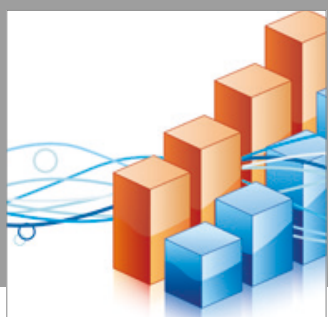

Advances in

Operations Research

vatersals

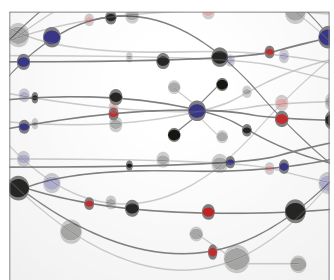

\section{The Scientific} World Journal
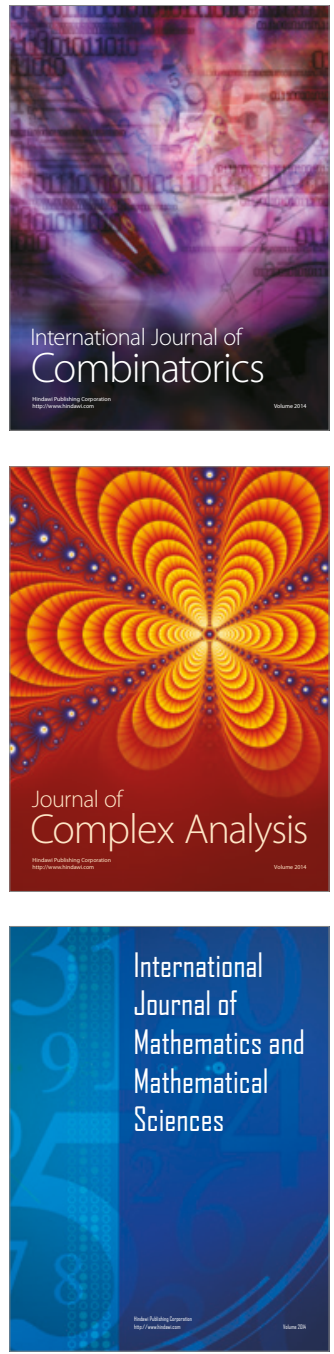
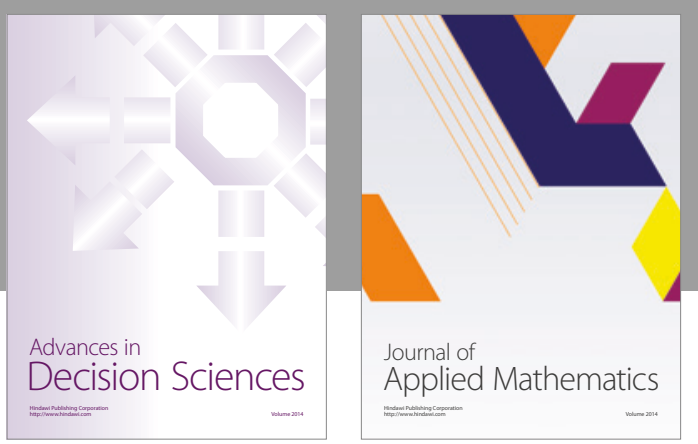

Algebra

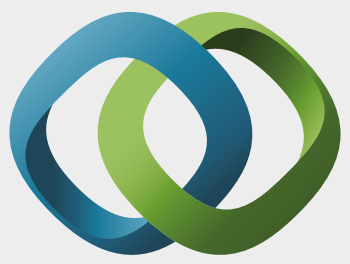

\section{Hindawi}

Submit your manuscripts at

https://www.hindawi.com
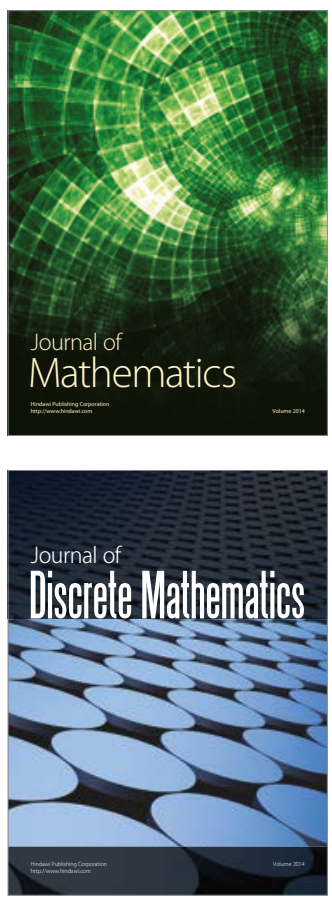

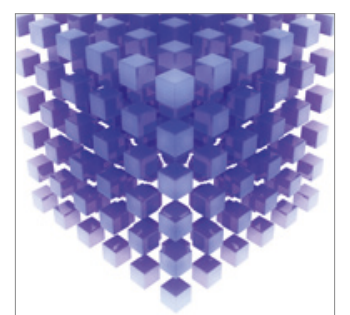

Mathematical Problems in Engineering
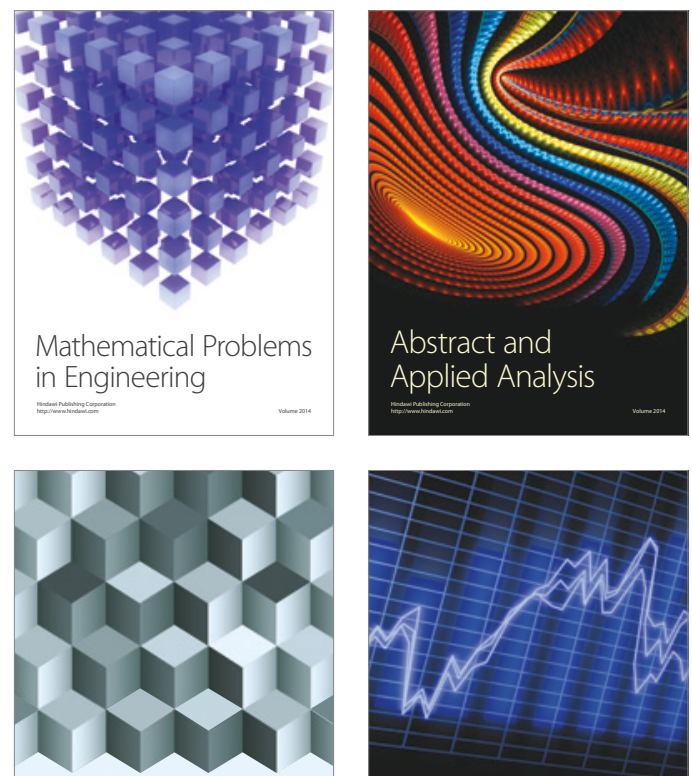

Journal of

Function Spaces

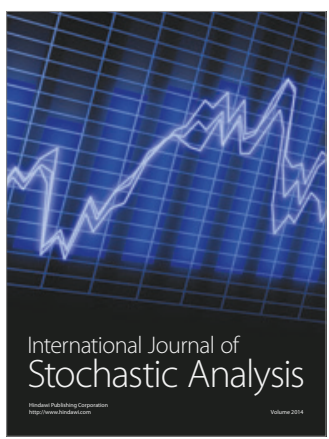

Probability and Statistics
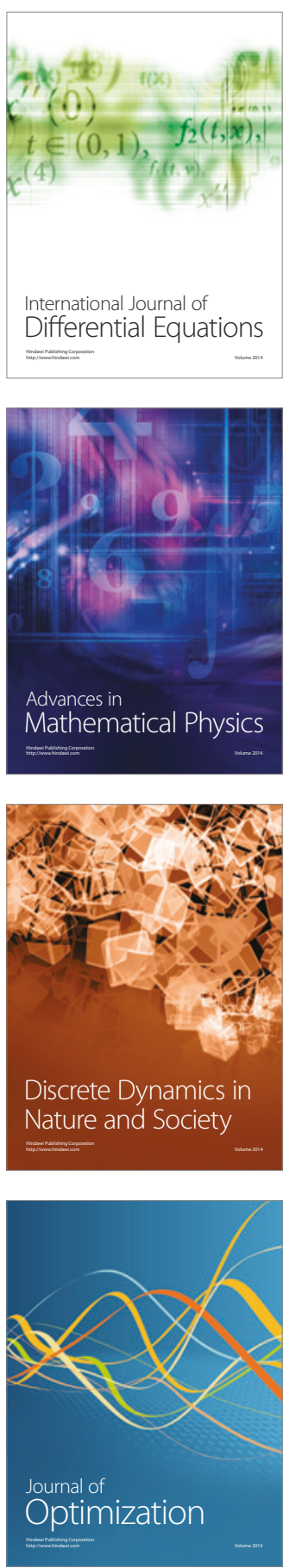